\title{
Response time analysis of hot-wire- based gas velocity sensor using optical fiber
}

Jiqiang Wang, Zhen Li, Tongyu Liu, Moyu Hou, Lin Zhao, et al.

Jiqiang Wang, Zhen Li, Tongyu Liu, Moyu Hou, Lin Zhao, Guofeng Dong, "Response time analysis of hot-wire-based gas velocity sensor using optical fiber," Proc. SPIE 11144, Photonics and Education in Measurement Science 2019, 1114418 (17 September 2019); doi: 10.1117/12.2531836

SPIE Event: Joint TC1 - TC2 International Symposium on Photonics and Education in Measurement Science 2019, 2019, Jena, Germany 


\title{
Response Time Analysis of 'Hot-wire'-based Gas Velocity Sensor Using Optical Fiber \\ Jiqiang Wang ${ }^{\text {a, b }}$, Zhen Li ${ }^{\mathrm{a}, \mathrm{b}}$, Tongyu Liu ${ }^{\mathrm{a}, \mathrm{b}}$, Moyu Hou ${ }^{\mathrm{a}, \mathrm{b}}$, Lin Zhao ${ }^{\mathrm{a}, \mathrm{b}}$ and Guofeng Dong ${ }^{\mathrm{a}, \mathrm{b}}$ \\ ${ }^{a}$ Laser Institute, Qilu University of Technology (Shandong Academy of Sciences), Jinan, Shandong,

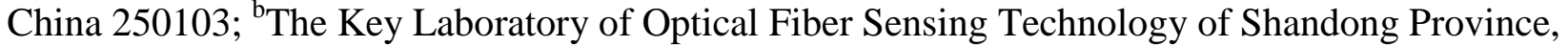 Jinan, Shandong, China 250103
}

\begin{abstract}
Built on a design developed from an advanced mathematical model, a practical fiber optic sensor, which is an analog of the familiar 'hot-wire' wind velocity monitor is developed, as an intrinsically-safe sensor device for coal mining monitoring applications. The underpinning optical fiber-based principle used is the shift in the center wavelength of a Fiber Bragg Grating which is cooled by the gas flowing over it and the device sensitivity found was determined to be $\sim 1370 \mathrm{pm}$ per unit $\mathrm{m} / \mathrm{s}$ wind velocity (in the range of $0-0.57 \mathrm{~m} / \mathrm{s}$ ), $\sim 109 \mathrm{pm}$ per unit $\mathrm{m} / \mathrm{s}$ in the range $0.57-2.26 \mathrm{~m} / \mathrm{s}$ and $\sim 33 \mathrm{pm}$ per unit $\mathrm{m} / \mathrm{s}$ in the range of $2.26-5.66 \mathrm{~m} / \mathrm{s}$. In this paper, the factors that influence the device response time, such as the sensor probe surface heat transfer coefficient, wind (gas) velocity and pump power have been investigated in the laboratory. It was found that the greater the surface dissipation factor of the sensor, the shorter the response time, furthermore, the response time was observed to decrease as the wind velocity increased. A method of further shortening sensor response time using wind speed variation slope is proposed.
\end{abstract}

Keywords: Fiber-optic gas velocity sensor; Hot-wire; Response time; Probe sensitivity

\section{INTRODUCTION}

Wind velocity measurement plays an important role in the fields of coal mine gas clearance, roadway ventilation and fire safety warning and is an important measurement parameter both in scientific research and many areas of industrial production. As sensors to measure gas/wind velocity are important for a number of such applications, considerable research has been carried out over the years into optimizing their design and performance, using a range of transduction techniques including fiber optic-based methods[1]. Most devices involve traditional electronic sensor methods, including volumetric measurement, turbines, differential pressure monitoring, ultrasonic measurements or electromagnetic flowmeters[2-5], for example. Intrinsic safety is required for applications such as coal mining and with the electrical methods mentioned amongst the above examples, there is the potential for 'spark hazards' which could cause explosions of flammable gases e.g. methane which can build up in mines. Optical fiber sensing technology offers the intrinsic safety required from an 'all-optical' measurement approach, at the low optical power levels used in these sensors, with the added benefit of the absence of electromagnetic interference. This allows the development of typically light weight, low power sensors which can readily be placed at considerable distances one from another in a mine (which may be of several kilometers in length) and thus may be sited where flammable gases and explosion hazards can occur[6].

The 'hot-wire' anemometer (HWA) is an important type of device that has previously been extensively investigated[7,8] as a familiar electrical device and in its optical analog can be used to measure the wind speed on the basis of a similar principle to the electrical 'hot wire' anemometer. The underpinning principle is that heat is lost to the air flowing over the heated active sensor element, and this is then related to the flow of gas over the heated element (the 'hot wire') and calibrated against the gas velocity. Such a device can be made very sensitive to low wind speeds, such as are experienced in coal mines and thus to be well suited to the application under consideration.

The research on 'Hot-wire'-based velocity sensor using optical fiber has started from 2005. In summary the previous studies[9-15], whilst having shown progress over a number of years, have mainly achieved this by including modifications to the fiber geometry, and having a mismatched fusion coupling of a multimode fiber to a single-mode fiber to form a FP cavity, together with fiber gratings inscribed in a cobalt-doped fiber, as well as using a range of coatings and an unusual fiber metal filling method. However, they still show several problems when applied to the design of a better and more effective fiber optic 'hot wire' sensor for the mining application that is the subject of this work. Furthermore, most of the work reported to date on fiber optic hot wire anemometers has been focused on research into new methods for improving the conversion efficiency of light to heat, as well as employing better temperature 
compensation methods. In this paper, the fiber-optic hot-wire sensor, focusing on the factors that affect its sensitivity and response time. A probe device, based on this, has been constructed and its performance assessed in light of operation in demanding conditions of coal mines.

\section{SENSING PRINCIPLE AND MATHEMATICAL MODEL}

The underlying approach to the sensor design is based on the heating of an optical fiber by light from a laser beam, and its cooling by the gas flowing over it (in this application, air) and where the gas/wind velocity is to be measured. The basic measurement principle analyzed is of a heated optical fiber which is connected to a heat-insulating material where the heated fiber is then cooled by the mass of the gas blowing over it, allowing the wind velocity to be measured. The heat in the fiber is generated by using the photothermal conversion effect in the (cobalt-doped) fiber, and this is given by the factor $Q$ in the model. In the analysis carried out, it is assumed that one part of this, given by $Q_{1}$, is trapped inside the sensor element to increase the internal energy and the remaining part, $Q_{2}$, is used to exchange heat with the outside fluid (through the air flow). Therefore

$$
Q=Q_{1}+Q_{2}
$$

The heat conduction loss, $Q_{\text {cond }}$, the convection loss, $Q_{\text {conv }}$, and the heat radiation loss, $Q_{\text {radi }}$, are now considered. The radiation heat transfer, $Q_{\text {radi }}$, is relatively small compared to the convective heat transfer, $Q_{\text {conv }}$, (ignoring the influence of radiative heat transfer), so the equation $Q=Q_{1}+Q_{\text {conv }}$ represents a suitable heat transfer model with an internal heat source, where the derivative differential equation that represents the situation is given by[16]:

$$
Q^{\prime}=\rho c V \frac{\partial t}{\partial \tau}+h A\left(t-t_{0}\right)
$$

Here $Q$ ' is the time derivative of $Q$, the factors $\rho, c, V, h$ and $A$ indicate the density, specific heat capacity, volume, heat convection heat transfer coefficient and heat dissipation area of the sensing element respectively, $t$ indicates the real-time temperature and $t_{0}$ is the initial temperature, where $\tau$ represents time in the equation.

When the sensor reaches a steady state equilibrium under a particular power level applied from the optical source, the temperature rises (and is assumed to be $t_{\mathrm{w}}$ ), so the following formula for $Q^{\prime}$ can be obtained and is given by:

$$
Q^{\prime}=-h A\left(t_{w}-t_{0}\right)
$$

When the sensor is placed in the air flow whose velocity is to be measured, the temperature gradually decreases as the wind speed increases and this situation is given by:

$$
\frac{\theta}{\theta_{0}}=\frac{t_{w}-t}{t_{w}-t_{0}}=e^{-\frac{h A}{\rho c V} \tau}
$$

where $\theta=t_{\mathrm{w}}-t$ represents the difference between the sensor temperature in real time and the steady-state temperature ultimately achieved, $\theta_{0}=t_{\mathrm{w}}-t_{0}$ indicates the maximum temperature difference achieved when the sensor is in use.

When the sensor is placed in the flow field and the air velocity gradually decreases, the temperature gradually rises, as is given by Equation (5).

$$
\frac{\theta}{\theta_{0}}=\frac{t-t_{0}}{t_{w}-t_{0}}=1-e^{-\frac{h A}{\rho c V} \tau}
$$

In the above equation, $t_{0}$ indicates the initial temperature, the quantity — is the time constant, which is denoted by $\tau_{\mathrm{c}}$. From the above analysis, it can be seen that the relationship between the temperature and the time during which the heated element is heated or cooled is shown in Figure 1. 


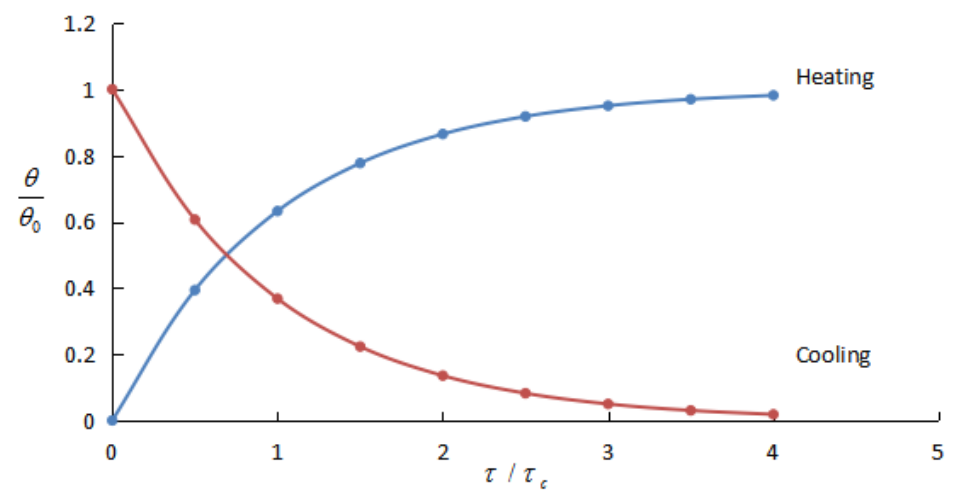

Figure 1. The relationship between temperature and the heating (blue) and the cooling (red) times

From the above figure, it can be seen that as the wind velocity increases and then decreases (that is when $\theta / \theta_{0}$ is equal to $63.2 \%$ and $36.8 \%$ respectively), the time $\tau$ associated with that is defined as the response time constant and expressed as $\tau_{\mathrm{c}}$.

When the sensor reaches a steady-state equilibrium, the temperature no longer changes, and the heat generated by the internal heat source is equal to the amount of heat lost, and hence:

$$
Q=h A \Delta t
$$

Thus for the Fiber Bragg Grating (FBG) in the optical fiber which is affected by this thermal effect, there is a corresponding wavelength change given by:

$$
\lambda=\lambda_{0}+k \Delta t
$$

where $\lambda$ is the FBG wavelength after the heating/cooling process is applied, $\lambda_{0}$ is the original FBG wavelength (under ambient conditions) and $k$ is the Fiber Bragg Grating temperature coefficient. When using this approach in an actual sensor, a simpler heat dissipation formula, King's law, can be used where:

$$
N_{u}=A_{c}+B_{c} R_{e}^{\frac{1}{2}}
$$

In this formula, $N_{\mathrm{u}}$ is the Nusselt number, $A_{\mathrm{c}}$ and $B_{\mathrm{c}}$ are constants that depend on the state of the fluid, and $R_{\mathrm{e}}$ is the Reynolds number[16] of the fluid.

Based on the above formula, the expression for the sensor dynamic range (which corresponds to the change in the wavelength of the FBG used), $\Delta \lambda$, and the time, $\tau$, is given by:

$$
\left\{\begin{array}{c}
\Delta \lambda=\frac{k v^{\frac{1}{2}} l_{2} Q}{k_{w}\left(A_{c} v^{\frac{1}{2}}+B_{c} l_{2}^{\frac{1}{2}} u^{\frac{1}{2}}\right) A} \\
\tau=\frac{\rho c V v^{\frac{1}{2}} l_{2}}{\left(A_{c} v^{\frac{1}{2}}+B_{c} l_{2}^{\frac{1}{2}} u^{\frac{1}{2}}\right) A}
\end{array}\right.
$$

In this formula, $v, u$, and $k_{\mathrm{w}}$ is the kinematic viscosity coefficient, the velocity and the thermal conductivity of the fluid respectively. $l_{2}$ is the characteristic length of the sensing element. It can be seen from the above equations that the wavelength change experienced by the FBG and the response time of sensor are both proportional to the heat capacity of the copper tube and also inversely proportional to the speed and heat dissipation area of the bushing used. 


\section{3. 'HOT-WIRE' BASED FIBER OPTIC WIND VELOCITY SENSOR DESIGN}

Based on the above analysis, a practical fiber-optic 'hot-wire' sensor probe was designed and built, following which its performance was evaluated. The design, shown in Figure 2, uses the principles established in the analysis and consists of a Fiber Bragg Grating (FBG) in one fiber, a further co-doped fiber, a bushing and a thermally insulated base. The FBG and the co-doped fiber are inserted, side-by-side, into a 'packaging' designed for the purpose and consisting of an outer copper tube, with an inner diameter of $0.6 \mathrm{~mm}$ and a wall thickness of $0.1 \mathrm{~mm}$. This is filled with silicone oil to allow good thermal conduction and thus to form the complete sensing element. One end of this is then brazed to the heat insulating material (to reduce the heat loss caused by the heat conduction of the sensing element) and this design improves the device sensitivity. The modelling carried out and reported above shows that the thermal resistance in the sensor element is much smaller than the convection thermal resistance and enables the correspondence between the wind speed and the center wavelength change of the FBG to be estimated. The sensor probe has the advantage of being relatively small, of low thermal mass and simple in structure, making it relatively inexpensive and convenient to use in different applications.

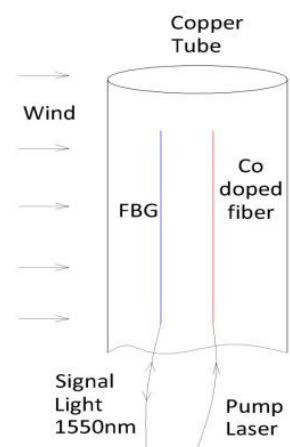

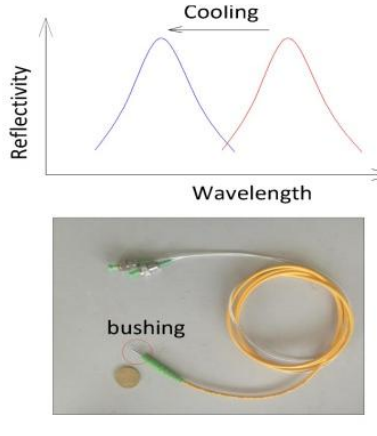

(a)

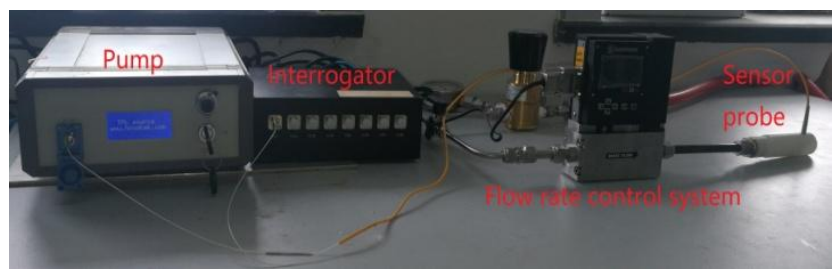

(b)

Figure 2. Schematic of the sensor probe (left), the FBG wavelength change with heating (red) and cooling (blue) (top) and a photograph of the sensor probe showing its small size and compact packaging. (a) Schematic and (b) Experimental device

\section{EXPERIMENTAL EVALUATION OF RESPONSE TIME}

The fiber optic 'hot wire' sensor system used is shown schematically in Figure 3. The range of available speeds from the flow rate control system is $0-10 \mathrm{~m} / \mathrm{s}$, (with an accuracy of $(0.03 \pm 0.7 \mathrm{Vmeasure}) \mathrm{m} / \mathrm{s}$, and the repeatability being $0.01 \mathrm{~m} / \mathrm{s}$. The pump light source operates at wavelengths of either 1480 or $1550 \mathrm{~nm}$, with the laser power being available over a range, up to $500 \mathrm{~mW}$. A commercial fast fiber grating interrogator of accuracy $\pm 2 \mathrm{pm}$, and repeatability of $2 \mathrm{pm}$, operating at a frequency of $1 \mathrm{kHz}$ is used to determine the wavelength shift in the grating, $\Delta \lambda$. The pump light source is connected to the co-doped fiber and the interrogator to the fiber with the FBG, both in the 'packaged' sensor probe. In operation, the co-doped fiber converts the input light energy into heat energy from the laser pump source, allowing the temperature around the FBG to rise and its central wavelength shifts towards the red, (the magnitude of the shift depending on the power applied). Then convection-based heat transfer occurs when the fluid flows through the bushing surface, heat is dissipated, the temperature around the FBG decreases, and its central wavelength shifts in the opposite direction (towards the blue) as the probe cools.

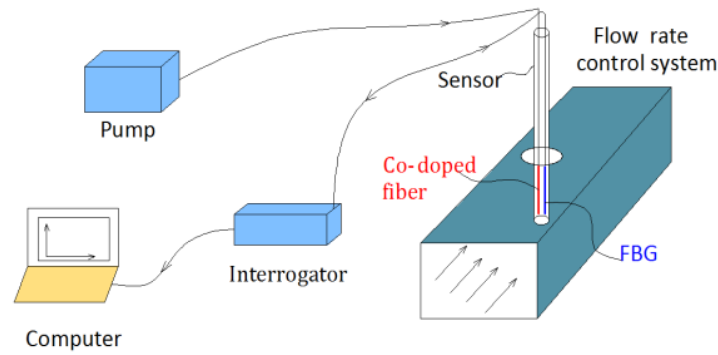

Figure 3. Schematic of the fiber-optic 'hot-wire' sensing system showing the laser pump, the demodulator/FBG interrogation system, the probe placed in a wind tunnel for testing and evaluation and the computer where the outputs are visualized 


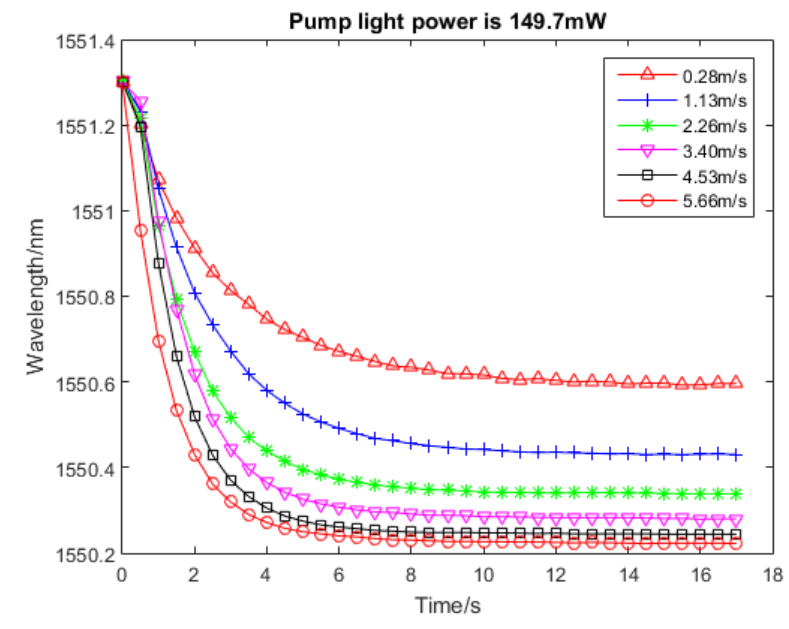

(a)

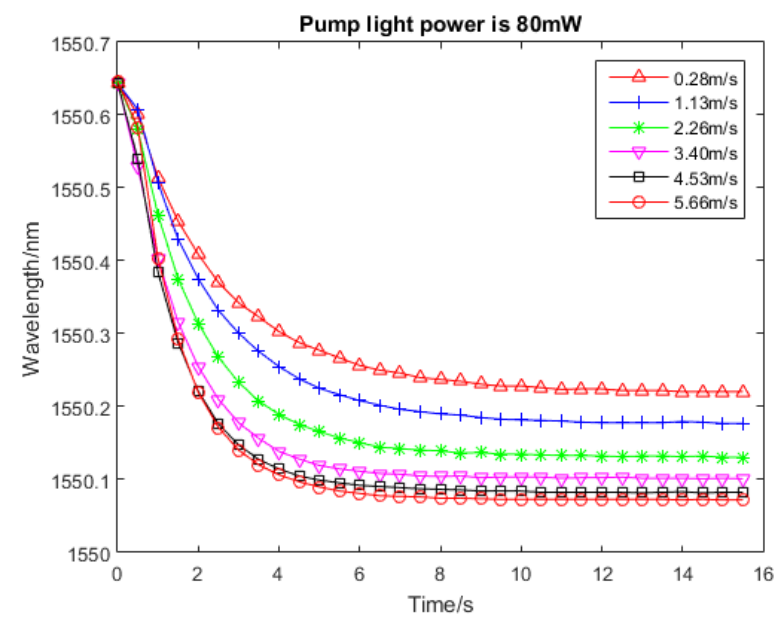

(b)

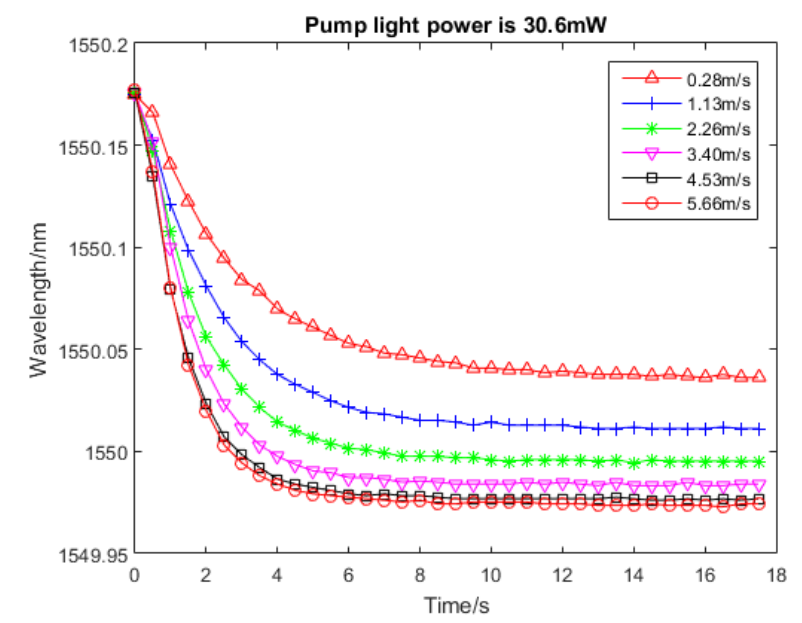

(c)

Figure 4. Measurement of the response time of a probe tested, monitoring the change in the FBG wavelength with time, each for a series of different wind velocities (as shown in the inset). (a) $149.7 \mathrm{~mW}$, (b) $80 \mathrm{~mW}$, (c) $30.6 \mathrm{~mW}$

To evaluate their performance, the probe with a pump light power of $149.7 \mathrm{~mW}, 80 \mathrm{~mW}, 30.6 \mathrm{~mW}$ used, were subjected to air flows at different (and known) velocities in the test wind tunnel. The sensitivity of the sensor is described through the results seen in Figure 4. From the results obtained as Figure 4(a), it can be seen that with the increase of wind speed, the FBG wavelength change observed is non-linear, with a higher sensitivity to wind velocity in the lower velocity range. If a commercially available wavelength interrogation device with a $1 \mathrm{pm}$ precision is used to extract the measurement data, the resolution of the sensing system is given from the figure as $\sim 1370 \mathrm{pm} /(\mathrm{m} / \mathrm{s})$ when the wind speed is in the range of 0 $0.57 \mathrm{~m} / \mathrm{s}, \sim 109 \mathrm{pm} /(\mathrm{m} / \mathrm{s})$ in the range of $0.57-2.26 \mathrm{~m} / \mathrm{s}$, and $\sim 33 \mathrm{pm} /(\mathrm{m} / \mathrm{s})$ beyond that (up to the maximum measured 5.66 $\mathrm{m} / \mathrm{s}$ in this work). The sensitivity of the device in the important $2.66-5.66 \mathrm{~m} / \mathrm{s}$ range is $33 \mathrm{pm} /(\mathrm{m} / \mathrm{s})$ thus the device is well suited to the wind tunnel facilities in which it was tested and to practical measurements in the mining applications considered.

Experiments were carried out to evaluate response time of a sensor probe under different wind speeds and at the same ambient temperature (to allow a close comparison), as shown in Figure 4 (a) (for $149.7 \mathrm{~mW}$ ), 4 (b) (for 80mW) and 4 (c) 
(for $30.6 \mathrm{~mW}$ ) respectively. where the wavelength shift caused by the different wind velocities used is monitored with time, until a steady state change is reached.

As can be seen from Figure 4, as expected the response times of the sensor probe decrease with increasing wind velocity and the wavelength dynamic range increases. The effect on the FBG wavelength change (for each wind velocity shown) is nonlinear with time. In summary, At the velocity of $0.28 \mathrm{~m} / \mathrm{s} \sim 5.66 \mathrm{~m} / \mathrm{s}$ and the pump light power of $149.7 \mathrm{~mW}, 80 \mathrm{~mW}$, $30.6 \mathrm{~mW}$, the FBG wavelength shift reaches $63 \%$ of the maximum wavelength change seen within 2.5 seconds. This sensor probe has a faster response time than the previous generation test prototype[17].

Through analyzing the wavelength change data at $80 \mathrm{~mW}$, it can be found that the first five data of each curve in Figure. 4(b) has good linearity, and the fitting curve slopes at different velocity is shown as Table 1 . Which are linearly correlated with the test velocity, shown as Figure 5. So a method to shorten the response time could be developed according to the above linear correlation.

Table 1. The first five data's slope of wavelength change curve at different wind velocities

\begin{tabular}{|c|c|}
\hline Velocity $(\mathbf{m} / \mathbf{s})$ & Slope of wavelength change \\
\hline 0.57 & -0.1235 \\
\hline 1.13 & -0.143 \\
\hline 2.26 & -0.1729 \\
\hline 3.40 & -0.1985 \\
\hline 4.53 & -0.2194 \\
\hline 5.66 & -0.2479 \\
\hline
\end{tabular}

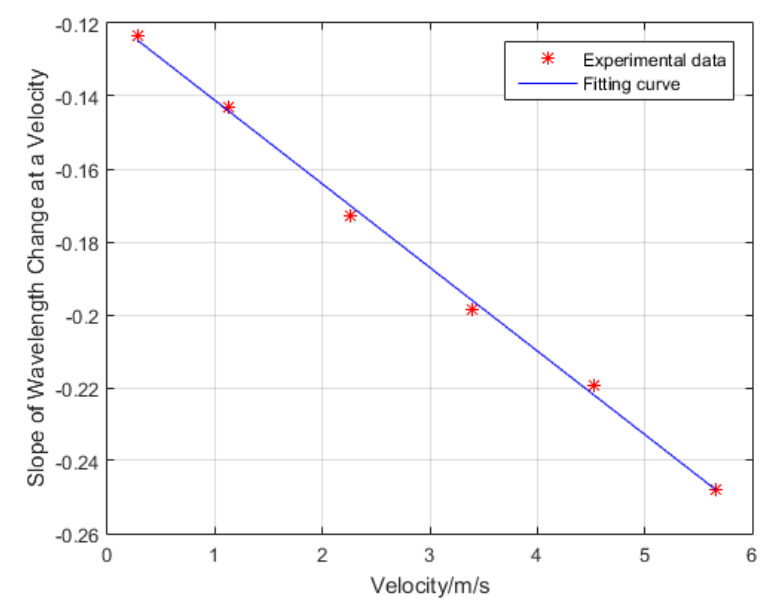

Figure 5. The first five data's slope of each wavelength change curve at the pump power of $80 \mathrm{~mW}$ and different velocity, and their fitting curve.

\section{DISCUSSION}

Using the principle of the 'hot-wire' anemometer, combined with optical fiber sensing phenomena, this paper has reported on both a theoretical analysis and the subsequent results of experiments carried out with several different probe designs for wind velocity measurement. Theoretical analysis shows that the dynamic range and sensitivity of the designs are related to the power of the light source used, the wind velocity and the heat transfer coefficient of the 'hot-wire' sensor package. The response time of the probe is shown to be related to the heat capacity of the packaging material, the wind speed, and the heat dissipation area. The results show that the fiber-optic 'hot-wire' wind velocity sensor is particularly sensitive at low speeds and has excellent performance. A method of further shortening sensor response time 
using wind speed variation slope is proposed. Work is on-going to improve the device, where the next step will be to study how to shorten its response time based on the method and increase its upper limit of measurement.

\section{REFERENCES}

[1] Basarab, I., Ghassemlooy, Z., Shaw, A. J., \& Grattan, K. T. V. (1991). A fluorescence-based fiber-optic flow sensor - Design considerations. Review of Scientific Instruments, 62(5), 1321-1325.

[2] Laghrouche, M.; Adane, A.; Boussey, J.; Ameur, S.; Meunier, D.; Tardu, S. A miniature silicon hot wire sensor for automatic wind speed measurements. Renew. Energy 2005, 30, 1881 - 18966.

[3] Newkirk, A.V.; Antonio-Lopez, J.E.; Salceda-Delgado, G.; Piracha, M.U.; Amezcua-Correa, R.; Schülzgen, A.Multicore fiber sensors for simultaneous measurement of force and temperature. IEEE Photonic Technol. Lett.2015, 27, 1523 - 1526.

[4] Firth, J.; Ladouceur, F.; Brodzeli, Z.; Wyres, M.; Silvestri, L. A novel optical telemetry system applied to flowmeter networks. Flow Meas. Instrum. 2016, 48, 15 - 19.

[5] Liu, G.G.; Hou, W.L.; Qiao, W.; Han, M. Fast-response fiber-optic anemometer with temperature self-compensation. Opt. Express 2015, 23, 13562 - 13570.

[6] G. Liu, M. Han, and W. Hou, High-resolution and fast-response fiber-optic temperature sensor using silicon FabryPérot cavity[J]. Opt. Express 23, 7237-7247 (2015).

[7] Takagi, S. A hot-wire anemometer compensated for ambient temperature variations. J. Phys. E Sci. Instrum.1986, $19,739-743$.

[8] Chen, T.; Wang, Q.; Zhang, B.; Chen, R.; Chen, K.P. Distributed flow sensing using optical hot-wire grid.Opt. Express 2012, 20, $8240-8249$.

[9] Cashdollar L J, Chen K P. Fiber Bragg grating flow sensors powered by in-fiber light[J]. IEEE Sensors Journal, 2005, 5(6):1327-1331.

[10] Jewart C, Mcmillen B, Cho S K, et al. X-probe flow sensor using self-powered active fiber Bragg gratings[J]. Sensors \& Actuators A Physical, 2006, 127(1):63-68.

[11] Caldas P, Jorge P A, Rego G, et al. Fiber optic hot-wire flowmeter based on a metallic coated hybrid long period grating/fiber Bragg grating structure[J]. Applied Optics, 2011, 50(17):2738-43.

[12]Zhang A P, Lu C, Tam H Y, et al. All-optical fiber anemometer based on laser heated fiber Bragg gratings[J]. Optics Express, 2011, 19(11):10124-30.

[13] Wang X, Dong X, Zhou Y, et al. Hot-Wire Anemometer Based on Silver-Coated Fiber Bragg Grating Assisted by No-Core Fiber[J]. IEEE Photonics Technology Letters, 2013, 25(24):2458-2461.

[14]Zhou B, Jiang H, Lu C, et al. Hot Cavity Optical Fiber Fabry-Perot Interferometer as a Flow Sensor With Temperature Self-Calibrated[J]. Journal of Lightwave Technology, 2016, 34(21):5044-5048.

[15]Zhang Y, Wang F, Duan Z, et al. A Novel Low-Power-Consumption All-Fiber-Optic Anemometer with Simple System Design[J]. Sensors, 2017, 17(9).

[16] Yang Shiming, Tao Wenshuan. Heat Transfer[M]. High Education Press, 2006,8.

[17]Zhen Li, Jiqiang Wang, Tongyu Liu, et al. High-Sensitivity "Hot-Wire"-Based Gas Velocity Sensor for Safe Monitoring in Mining Applications [J]. IEEE Sensors Journal, 2018, 18 (24): 10192-10198 